\title{
Use of mineralocorticoid receptor antagonist in ST elevation myocardial infarction
}

\author{
Muhammad H. Khan, MD, and Myron C. Gerson, MD $^{\mathrm{b}, \mathrm{c}}$ \\ a University of Cincinnati Department of Internal Medicine, Cincinnati \\ b University of Cincinnati Department of Cardiovascular Medicine, Cincinnati \\ c Division of Cardiovascular Health and Disease, Department of Internal Medicine, University of \\ Cincinnati College of Medicine, Cincinnati, $\mathrm{OH}$
}

Received Aug 22, 2021; accepted Aug 23, 2021

doi: $10.1007 / \mathrm{s} 12350-021-02801-9$

\section{See related article, pp. 2325-2335}

\section{INTRODUCTION}

Cardiovascular deaths are the leading cause of mortality worldwide, and the majority of these deaths are associated with coronary ischemic diseases. A significant reduction in morbidity and mortality has been observed with increasingly accessible reperfusion therapy after acute ST elevation myocardial infarction (STEMI). The AHA/ACC guidelines recommend smoking cessation, aggressive lipid lowering, control of hypertension and diabetes, and prophylactic use of aspirin, beta-blockers, and angiotensin-converting enzyme inhibitors (ACE-I) for secondary prevention after STEMI. ${ }^{1}$ The use of a mineralocorticoid receptor antagonist (MRA) is only recommended in patients receiving therapeutic doses of ACE-I, demonstrating a left ventricular ejection fraction $\leq 40 \%$, and having either diabetes or heart failure. ${ }^{1}$

In this issue of the Journal, a study by Toda et $\mathrm{al}^{2}$ demonstrates the effects of MRAs on cardiac sympathetic innervation in post-STEMI patients. It raises the potential benefit of MRAs in reducing left ventricular remodeling in post-STEMI patients without heart failure and who otherwise do not meet the therapeutic criteria for MRA treatment as per the Eplerenone Post-Acute

Funding None.

Reprint requests: Myron C. Gerson, MD, Division of Cardiovascular Health and Disease, Department of Internal Medicine, University of Cincinnati College of Medicine, 231 Albert Sabin Way, Cincinnati, OH 45267; gersonml@ucmail.uc.edu

J Nucl Cardiol 2022;29:2336-9.

$1071-3581 / \$ 34.00$

Copyright (c) 2021 American Society of Nuclear Cardiology.
Myocardial Infarction Heart Failure Efficacy and Survival Study (EPHESUS) trial. ${ }^{3}$ Previous studies have elucidated the positive effects of MRAs such as eplerenone and aldosterone in heart failure, but their effect on improving cardiac sympathetic tone and preventing cardiac remodeling after an acute STEMI is not well established.

\section{DISCUSSION}

\section{Use of MIBG After STEMI}

Iodine-123-metaiodobenzylguanidine (MIBG) is a radiopharmaceutical that depicts the integrity and health of cardiac sympathetic innervation. MIBG has been investigated extensively for documenting the dysregulation of cardiac sympathetic nerve activity after an acute MI. An abnormally high washout rate (WR), high total defect score (TDS), or low heart-to-mediastinum ratio (HMR) on MIBG scintigraphy is associated with poorer long-term outcome. ${ }^{4}$ Additionally, studies have demonstrated a correlation between severity of postSTEMI cardiac denervation on MIBG with the size of the infarcted myocardial tissue and the severity of left ventricular dysfunction. ${ }^{5}$ However, routine use of MIBG after an acute STEMI for the purpose of cardiac sympathetic nervous system assessment is not a standard practice based on the current recommendations by the AHA/ACC. ${ }^{1}$

\section{Pathophysiology of Ventricular Remodeling}

Myocyte necrosis due to an acute STEMI triggers a cascade of molecular and mechanical processes that initiate ventricular remodeling pathways. Among the many adaptive pathophysiological changes, two important remodeling processes, that are often targets of drug 
therapies, are regulation of the cardiac sympathetic nervous system and the renin angiotensin aldosterone system. $^{6}$

\section{Effects of MRA on Cardiac Sympathetic Nerve Activity}

To understand how MRAs can prevent cardiac remodeling, it is important to review the effect of MI on the cardiac sympathetic nervous system.

First, immediately after an MI, there is a sudden and sustained increase in left ventricular loading conditions and cardiac sympathetic nerve activity due to perturbations in circulatory hemodynamics. This results in the release of catecholamines from the efferent sympathetic nerve terminals. This then augments cardiac contractility and heart rate, resulting in hyperkinesis of the noninfarcted myocardial tissue and temporary circulatory compensation. However, a sustained increase in cardiac sympathetic nerve activity exacerbates ischemic damage to the infarcted and peri-infarct tissue, worsening cardiac remodeling and impairing ventricular ejection fraction. ${ }^{6}$ Numerous murine and canine in vivo studies have documented that excessive norepinephrine results in increased intracellular calcium level, which is toxic to myocytes and induces pathways for apoptosis, resulting in ventricular dysfunction.

Neurohormonal activation, myocardial stretch, and activation of the renin angiotensin system all contribute to myocardial hypertrophy during a second, later phase of myocardial infarction (MI). The resultant eccentric myocardial hypertrophy further increases myocardial remodeling, resulting in reduced left ventricular ejection fraction. ${ }^{7,8}$ A further late sequela of acute $\mathrm{MI}$ is regional sympathetic denervation in the infarcted myocardium and the adjacent border-zone myocardial tissue. In patients with myocardial ischemia, the sympathetic nerves that run along the course of the occluded coronary artery on the epicardial surface of the heart are lost because of ischemic injury or scarring. At this stage of MI, cardiac denervation may have some protective effect, as it attenuates catecholamine overstimulation in a failing heart. This has been observed in rat models following stellate ganglionectomy. ${ }^{9}$ Similarly, a canine in vivo study demonstrated the protective effect of cardiac denervation on the development of heart failure by observing reduced left ventricular diastolic pressure and wall stress, and increased overall myocardial contractility. ${ }^{10}$ However, frequently, the extent and severity of myocardial sympathetic denervation extends beyond the infarcted tissue and tends to enhance ventricular dysfunction in otherwise normal ventricular myocytes. In fact, functional abnormality due to denervation of the non-infarcted adjacent myocardial tissue can contribute to a reduction in myocardial contractility by increased local wall stress and cellular hypertrophy that alters Frank-Starling kinetics and reduces the overall ventricular ejection fraction. ${ }^{11}$

The beneficial effects of MRA in patients with reduced left ventricular ejection fraction have been demonstrated in the RALES and EPHESUS trials., ${ }^{3,12}$ Central to the beneficial effects of MRA is its effect on the cardiac sympathetic nervous system. Aldosterone increases cardiac sympathetic nerve activity, which increases contractility of the failing heart (a cardinal response to heart failure). By preventing reuptake of norepinephrine and increasing adrenergic tone, aldosterone promotes structural remodeling, resulting in heart failure. MRAs counteract this process by blocking the effect of aldosterone, preserving norepinephrine reuptake at the synaptic cleft, and improving overall cardiac sympathetic nerve activity, as demonstrated by MIBG scintigraphy. HMR and WR are two main indices in MIBG scintigraphy, commonly used in evaluating the severity, prognosis, and treatment response of heart failure. Improvement in the HMR and WR with pharmacologic therapy has been documented predominantly in patients with heart failure related to cardiomyopathy. ${ }^{13-16}$ The present study extends those observations to patients with STEMI.

The present study by Toda et $\mathrm{al}^{2}{ }^{2}$ for the first time, studied the effect of eplerenone on cardiac sympathetic nerve activity and left ventricular remodeling in patients with acute STEMI but with no known symptoms of heart failure. The study demonstrated that initiating eplerenone in patients with first STEMI results in improved TDS, HMR, and WR indices compared to the group that did not receive eplerenone. Furthermore, the study demonstrated significantly increased left ventricular ejection fraction after 3 weeks of therapy in the eplerenone group, whereas the degree of change in left ventricular ejection fraction in the non-eplerenone group was significantly lower. The study, however, did not indicate whether the patients had developed symptoms of heart failure after STEMI and it excluded patients with severe heart failure requiring mechanical support. ${ }^{2}$ Important factors that need future exploration are the impact of the location of the culprit coronary artery and the size of the infarcted tissue. Feiring et al has reported a statistically significant association between the size of the area at risk and the reduction in the left ventricular ejection fraction after STEMI, and this may be a factor in the potential benefit of MRA administration. ${ }^{17}$ Further, in the study by Toda et al, TDS was calculated for the entire left ventricle, rather than that for the culprit coronary artery causing STEMI. 
The case for use of eplerenone in the treatment of STEMI in the absence of a substantially decreased global left ventricular ejection fraction is further bolstered in the present study by the observation that patients treated with eplerenone had evidence of reduced myocardial fibrosis, as assessed by procollagen type III $\mathrm{N}$-terminal peptide (PIIINP) assay, compared to untreated patients. These results are in accordance with the results of the REMINDER (early eplerenone treatment in patients with acute ST elevation MI without heart failure) and the RALES trials, demonstrating a reduction in extracellular matrix biomarkers such as PIIINP and procollagen type $1 \mathrm{~N}$-terminal propeptide with the use of MRAs, suggesting their role in limiting the adverse cardiac remodeling and improving survival and heart failure hospitalization rates. ${ }^{12,18}$

\section{CONCLUSION}

Post-MI therapies are best judged by their impact on clinical outcomes. MRAs have demonstrated survival benefit in patients with diabetes or heart failure who have a reduced left ventricular ejection fraction $(\leq 40 \%)$ after an acute STEMI. It is also known that MRA therapy after STEMI may predispose those patients to developing renal and electrolyte abnormalities. Use of MRAs in STEMI patients with no known symptoms of heart failure and/or minimal or no reduction in left ventricular ejection fraction needs to be explored in larger clinical trials with rigorous evaluation of their risks and benefits. In addition to other outcomes, the extension of eplerenone therapy to a lower risk STEMI population requires evaluation of the effect of treatment on alleviation of symptoms. Nevertheless, the present study provides direction for a potential therapeutic advance with eplerenone treatment by demonstrating improved sympathetic nerve function, reduced left ventricular fibrosis, and reduced left ventricular remodeling in a group of STEMI patients with mildly reduced or normal left ventricular function.

\section{Disclosures}

The authors have no conflicts of interest to declare.

\section{References}

1. Antman E, Anbe D, Armstrong P, Bates E, Green L, Hand M, et al. ACC/AHA guidelines for the management of patients with ST-elevation myocardial infarction-Executive summary. Circulation 2004;110:588-96.

2. Toda K, Kasama S, Toyama T, Kasahara M, Kurabayashi M. Effects of mineralocorticoid receptor antagonist eplerenone on cardiac sympathetic nerve activity and left ventricular remodeling after reperfusion therapy in patients with first ST-segment elevation myocardial infarction. J Nucl Cardiol 2021. https://doi.org/10. 1007/s12350-021-02733-4.

3. Pitt B, Williams G, Remme W, Martinez F, Lopez-Sendon J, Zannad F, et al. The EPHESUS trial: Eplerenone in patients with heart failure due to systolic dysfunction complicating acute myocardial infarction. Eplerenone Post-AMI Heart Failure Efficacy and Survival Study. Cardiovasc Drugs Ther 2001;15:79-87.

4. Kasama S, Toyama T, Sumino H, Kumakura H, Takayama Y, Minami K, et al. Prognostic value of cardiac sympathetic nerve activity evaluated by ${ }^{[1231]} \mathrm{m}$-iodobenzylguanidine imaging in patients with ST-segment elevation myocardial infarction. Heart 2011;97:20-6

5. McGhie A, Corbett J, Akers M, Kulkarni P, Sills M, Kremers M, et al. Regional cardiac adrenergic function using I-123 metaiodobenzylguanidine tomographic imaging after acute myocardial infarction. Am J Cardiol 1991;67:236-42.

6. Sutton MG, Sharpe N. Left ventricular remodeling after myocardial infarction: Pathophysiology and therapy. Circulation 2000;101:2981-8

7. Hall TM, Gordon C, Roy R, Schwenke DO. Delayed coronary reperfusion is ineffective at impeding the dynamic increase in cardiac efferent sympathetic nerve activity following myocardial ischemia. Basic Res Cardiol 2016;111:35.

8. Zhou M, Liu Y, Xiong L, Quan D, He Y, Tang Y, et al. Cardiac sympathetic afferent denervation protects against ventricular arrhythmias by modulating cardiac sympathetic nerve activity during acute myocardial infarction. Med Sci Monit 2019;25:198493.

9. Zhang M, Zhu P, Wang Y, Wu J, Yu Y, Wu X, et al. Bilateral sympathetic stellate ganglionectomy attenuates myocardial remodelling and fibrosis in a rat model of chronic volume overload. J Cell Mol Med 2019;23:1001-13.

10. Sato N, Vatner SF, Shen YT, Kudej RK, Ghaleh-Marzban B, Uechi M, et al. Effects of cardiac denervation on development of heart failure and catecholamine desensitization. Circulation 1997;95:2130-40.

11. Kramer CM, Nicol PD, Rogers WJ, Suzuki MM, Shaffer A, Theobald TM, et al. Reduced sympathetic innervation underlies adjacent noninfarcted region dysfunction during left ventricular remodeling. J Am Coll Cardiol 1997;30:1079-85.

12. Pitt B, Zannad F, Remme WJ, Cody R, Castaigne A, Perez A, et al. The effect of spironolactone on morbidity and mortality in patients with severe heart failure. Randomized Aldactone Evaluation Study Investigators. N Engl J Med 1999;341:709-17.

13. Kasama S, Toyama T, Kumakura H, Takayama Y, Ichikawa $S$, Suzuki T, et al. Effect of spironolactone on cardiac sympathetic nerve activity and left ventricular remodeling in patients with dilated cardiomyopathy. J Am Coll Cardiol 2003;41:574-81.

14. Barr CS, Lang CC, Hanson J, Arnott M, Kennedy N, Struthers AD. Effects of adding spironolactone to an angiotensin-converting enzyme inhibitor in chronic congestive heart failure secondary to coronary artery disease. Am J Cardiol 1995;76:1259-65.

15. Shinohara H, Fukuda N, Soeki T, Sakabe K, Onose Y, Tamura Y. Effects of angiotensin II receptor antagonists on $\left[{ }^{(123)} I\right]$ metaiodobenzylguanidine myocardial imaging findings and neurohumoral factors in chronic heart failure. Heart Vessels 2002;17:47-52.

16. Kasama S, Toyama T, Sumino H, Matsumoto N, Sato Y, Kumakura $\mathrm{H}$, et al. Additive effects of spironolactone and candesartan on cardiac sympathetic nerve activity and left ventricular remodeling in patients with congestive heart failure. J Nucl Med 2007;48:1993-2000. 
17. Feiring AJ, Johnson MR, Kioschos JM, Kirchner PT, Marcus ML, White CW. The importance of the determination of the myocardial area at risk in the evaluation of the outcome of acute myocardial infarction in patients. Circulation 1987;75:980-7.

18. Ferreira JP, Duarte K, Montalescot G, Pitt B, de Sa EL, Hamm $\mathrm{CW}$, et al. Effect of eplerenone on extracellular cardiac matrix biomarkers in patients with acute ST-elevation myocardial infarction without heart failure: Insights from the randomized double-blind REMINDER Study. Clin Res Cardiol 2018;107:4559 .

Publisher's Note Springer Nature remains neutral with regard to jurisdictional claims in published maps and institutional affiliations. 